\title{
Using Confluence Theory to Make Unusual Predictions about Desire, Expectation, and Intention Attributions
}

\author{
Stephen Rice \\ Florida Institute of Technology, USA \\ David Trafimow \\ New Mexico State University, USA \\ Rian Mehta \\ Florida Institute of Technology, USA
}

Received: October 28, 2015 Accepted: January 31, 2016 Published: February 1, 2016

doi: 10.5296/jsss.v3i2.8505

URL: http://dx.doi.org/10.5296/jsss.v3i2.8505

\begin{abstract}
The intention attribution literature suggests that a success or a failure by a skilled person will result in a large difference in the intentions others will attribute to that person, but that this effect will be attenuated dramatically if the person is unskilled. The received literature is less clear about what to predict for desire attributions or expectation attributions. Based on a recent theory, the confluence theory (Trafimow, 2009), we predicted very little attenuation for desire attributions and only moderate attenuation for expectation attributions. These findings were confirmed across six experiments. Together, the experiments suggest an attenuation continuum where the attenuation effect increases as attributions pertain to variables more proximal to actual behavior.
\end{abstract}

Keywords: Confluence, Theory, Intentions

\section{Introduction and Background}

In a series of papers (see Reeder, 2009a for a review), Reeder has advanced the attribution area by pointing out that people can form motive inferences, as well as trait inferences, and that motive inferences likely will influence trait inferences. For example, imagine an observer who notices that Amy picks up some objects dropped by a woman in a hallway. The observer 
could make the trait inference that Amy is "helpful." Alternatively, if the observer knows that the woman in the hallway is Amy's boss, the observer might make a motive inference that Amy was trying to ingratiate herself with her boss. In this latter case, it is less likely that the observer would make the trait inference that Amy is helpful and might be more likely to make the trait inference that Amy is "ingratiating." The example illustrates the importance of multiple inferences.

Reeder summarized his work under his Multiple Inference Model (MIM; Reeder, 2009a). In essence, Reeder argued that the combination of a person performing a behavior in a particular situation causes observers to infer that person's motive and, in turn, the motive inference likely will influence the trait inference. An exception would be if the observer perceives the behavior as unintentional, in which case it might seem less sensible to infer a motive. We later will return to intentionality.

In a comment on Reeder's (2009a) MIM model, Trafimow (2009) pointed out that although motive inferences can influence trait inferences, as MIM states, influence traverses the opposite direction too-from trait inferences to motive inferences. For example, suppose an observer notices that Pete's boss criticizes him for his work. An observer might make the attribution that Pete's work was not very good. But suppose the observer was well aware that Pete's boss greatly enjoys criticizing what others do and has the corresponding trait expectation. In that case, the observer might infer, from this general trait expectation, that Pete's boss had the motive to criticize. Thus, Trafimow suggested that inferences go not only from motives to traits but also in the reverse direction.

In addition, Trafimow (2009) pointed out how other factors (such as prior expectations about the situation or the target person, or the observer's own motives) can influence motive and trait attributions, thereby providing yet a third class of mechanisms by which multiple inferences can influence each other. Several commentaries (Ames, 2009; Gawronski, 2009; Hamilton, Way, \& Chen, 2009; Molden, 2009; Morris, 2009; Newman, 2009; Phillips, 2009; Read, 2009; Trafimow, 2009), as well as Reeder's well-considered response to them (Reeder, 2009b), suggest consensus that there are several mechanisms by which inferences can influence each other. All of these considerations suggest a more general notion that associated mental elements have a tendency to influence each other and to become more compatible with each other. Trafimow (2009) termed this notion the confluence theory.

The confluence theory suggests interesting consequences. For example, if there is a tendency for mental elements to become compatible with each other, then the effects of psychological constructs on other ones all should be reversible. The reversibility principle is somewhat similar to that of equilibrium in chemistry. For every reaction, there also is a reaction in the opposite direction, though it might be too small to be perceived easily. Equilibrium is reached when the rates of the reaction and opposite reaction are equal. Recent evidence supports the validity of this reversibility principle in research on just world phenomenon. Rice and Trafmow (2011) performed a set of experiments where participants were exposed to target person's who had various outcomes. Consistent with the just world phenomenon, target persons with better outcomes were judged to be better people. In addition, however, Rice and 


\section{Macrothink}

Trafimow varied the moral characteristics of target persons and participants made guesses about outcomes for them. Consistent with the reversibility principle, participants judged that morally superior target persons would have better outcomes.

As another example, Trafimow et al. (2012) performed a set of experiments concerned with the relations between beliefs about consequences of behaviors, evaluations of those beliefs, and attitudes towards behaviors. Much previous research supports that beliefs about the consequences of behaviors and evaluations of those consequences determine behaviors (e.g., see Fishbein \& Ajzen, 2010 for a review). However, in a complicated set of experiments, Trafimow et al. demonstrated a reverse effect. That is, although beliefs about the consequences of a behavior and evaluations of the behavior strongly influenced attitudes, there was a reverse effect whereby the attitudes thereby formed influenced the very beliefs and evaluations that had led to their formation.

Another implication of the confluence theory is that the mere association of two mental elements is sufficient for them to become more similar to each other, unless there is a counterforce. For example, Rice, Trafimow, Keller, and Bean (in press) performed a demonstration in the ergonomics area, in an experiment involving two gauges. One gauge was perfectly reliable whereas the other gauge was at $70 \%$ reliability. Participants were exposed to much data on the performance of each gauge, and the instructions were written so as to maximize the ability of participants to distinguish between the two gauges. Nevertheless, when participants were given the opportunity to agree or disagree with the gauges on large sets of trials, they failed to differentially trust the two gauges; their responses across the trials revealed a pull-down effect. That is, the loss of trust in the unreliable aid caused participants to lose trust in the reliable aid as well, harming performance in that corresponding gauge.

For an even more extreme demonstration, consider again the Trafimow et al. (2012) article concerning beliefs, evaluations of beliefs, and attitudes. In two of the experiments, these researchers presented participants with beliefs that were completely irrelevant to evaluations that they measured later. For example, participants were told that a target person was honest or they were told that the target person was honest and had other positive traits too. The task was to decide whether to vote for the target person. Although the number of positive traits attributed to the target person is relevant to judging him, this number is not relevant to how good it would be to have a more honest government. In more general terms, beliefs about a target person are not logically relevant to evaluations of governmental consequences but participants nevertheless made the connection. Furthermore, in a final experiment, Trafimow et al. ruled out potential alternative explanations, and showed that this effect was caused by participants forming mental associations between beliefs about the target person and the positivity or negativity of governmental consequences, instigated by actually having to make a voting decision. Making the voting decision induced participants to form associations that they normally would not have made, the mental elements thereby associated became compatible with each other, and the result was the "irrelevancy effect" that Trafimow et al. obtained.

To understand the present extension of the confluence notion, consider the physical analogy 
of Newton's law of gravitation as it applies to an apple and the Earth. The Earth and the apple attract each other and tend to accelerate towards each other. However, it is possible to counteract this tendency by placing the apple on a table; the table provides a counterforce to the gravitational field. Returning to psychology, although general confluence implies that associated mental elements become increasingly compatible over time, there can be counterforces capable of attenuating, stopping, or even reversing the effect. Suppose, for example, that elements $A, B$, and $C$ are associated with $X$. In addition, suppose there exists a strong counterforce pertaining to the $X$ - $A$ relation, a weak counterforce pertaining to the $X$ - $B$ relation, and no counterforce pertaining to the $X$ - $C$ relation. Well, then, $X$ and $A$ should not become compatible, $X$ and $B$ should become somewhat compatible, and $X$ and $C$ should become very compatible. At present, the confluence theory implication that the tendency for mental elements to become compatible is qualified by the relevant counterforces has not been specifically explicated nor has it been shown to make any surprising predictions that contradict the predictions of another theory. Our goal is to fulfill both of these aims.

\subsection{Desire, Expectation, and Intention Attributions}

As summarized by Reeder (2009a), several philosophers and social psychologists have argued that various conditions are necessary for people to attribute intentionality when a target person performs a behavior. The target person must not only desire a particular outcome but he or she also must believe or expect that certain consequences are likely to arise (and these thoughts must have occurred prior to the behavior). Furthermore, the target person must have intended (chosen, decided, etc.) to perform the behavior and also must have been aware of performing it while doing so. There also is evidence for a fifth condition; the target person must have the requisite skill to perform the behavior (Mele \& Moser, 1994). Much evidence supports that, even when participants agree that the target person "wanted" to perform the behavior of concern, performance of the behavior was much more likely to be deemed "intentional" when the other conditions were met (e.g., Malle, 1999; 2004).

It is possible to make predictions from this received view, though the predictions differ depending on which auxiliary assumptions one includes. To see this, suppose that participants are exposed to skilled or unskilled persons who succeed or fail at a task. Consider first the case of failure. In this case, there is no strong reason to attribute intention in either the high skill or low skill condition, though the skilled person who failed might be assumed to have had a particularly low level of intention or even a negative intention (an intention to fail). In the case of success, because skill is a prerequisite for participants to attribute an intention, intention attributions should be strongly made in the high skill condition and attenuated in the low skill condition.

But suppose that participants are asked for desire attributions rather than for intention attributions. What would the received view predict? To commence, consider that the received view is that although a desire to perform a behavior is a precondition for an observer to attribute an intention to perform it, an intention to perform the behavior is not a precondition for an observer to attribute desire. Desire attributions are not supposed to depend on skill (Malle, 1999; 2004; Reeder, 2009a), though we can see the possibility of predicting effects 
anyway. An argument can be made that people might assume more effort (and more desire) in the case of success than in the case of failure and so desire attributions should be greater in the former case than in the latter one (Reeder et al., 2001). An argument also can be made that, in the case of failure, the high skill person must not have desired it or else he or she would have succeeded. Before continuing, we wish to be clear that we are not assuming that desire and effort attributions always go together. It is easy to imagine situations where a person desires something impractical or unethical and so it would be unlikely for the person to put forth much effort to fulfill that desire. However, if a person does put forth effort to succeed at a task, it seems reasonable to suppose that the person desired to succeed.

This reasoning implies more negative desire attributions for the high skilled person than for the low skilled person in the case of failure. What should be the prediction in the event of success? It seems obvious that it should be harder for the low skill person to succeed than for the high skill person to succeed and so success would seem to imply more desire on the part of the low skill person than the high skill person (Reeder et al., 2001). In summary, the received view predicts more negative desire attributions for skilled than unskilled persons in the event of failure and more positive desire attributions for unskilled than skilled persons in the event of success.

The received view can suggest a different prediction with the addition of different auxiliary assumptions. Suppose we make an auxiliary assumption that skilled persons are able to acquire desired outcomes whereas unskilled persons are not. In that case, desires and outcomes should be highly associated with each other in the condition of high skill but not in the condition of low skill. Thus, if the target person is skilled, success implies desire and failure implies lack of desire or negative desire; but if the target person is unskilled, there are no implications for desire. Therefore, as is predicted for intention attributions, success or failure also should greatly influence desire attributions pertaining to skilled persons but this effect should attenuate strongly for desire attributions pertaining to unskilled persons.

The confluence theory suggests the possibility of a "silly" prediction pertaining to desire attributions that differs from any of the foregoing predictions. To see why, consider the following reasoning. Let us assume that desire, expectation, and intention all are associated with the outcome. In this case, if the outcome is a success, then these variables all should be assumed to be positive and if the outcome is a failure then this should not be so. Thus, desire, expectation, and intention all should become compatible with the outcome. In the case of intentions, however, the fact that skill is considered to be a prerequisite for intention provides a counteracting force. Thus, predictions about intention attributions are similar from the confluence theory perspective and from the received view; the difference in intention attributions following success or failure should be attenuated in the poor skill condition. But now let us consider desire attributions. We assume that skill is not nearly as strongly associated with desire as it is with intentions. Therefore, there should be little to counteract the general tendency towards compatibility that the confluence theory assumes. In turn, this implies that skill level should be much less important for desire attributions than for intention attributions. Put another way, we expect that success will "engulf the field" for desire attributions, with relatively little in the way of qualifying effects of skill level. 


\section{Macrothink}

This seemingly silly prediction can be enhanced slightly with additional reasoning. We mentioned earlier the possibility that if a person succeeds, it might suggest that more effort may have been necessary in the low skill than in the high skill condition, and so the success implies more desire in the former than in the latter condition. In contrast, the confluence theory suggests that because skill and desire are not strongly associated, there should be very little in the way of qualifying effects of skill. Furthermore, what little effect there might be should be in the opposite direction from that proposed by the foregoing view. That is, the three-way combination of success, high skill, and high desire provides for more overall compatibility than the three-way combination of success, low skill, and high desire. Because the desire attribution is the only variable that is under participant control, there should be a small tendency for desire attributions to be greater when high skill is paired with success than when low skill is paired with success. Hence, we have a derivation of the prediction that desire attributions should be slightly enhanced when a skilled person succeeds compared to when an unskilled person does.

The foregoing discussion can be summarized easily with the following statistical hypotheses. When intention attributions are made, there should be a main effect for the outcome (success or failure) but it should be qualified by an interaction with the person's level of skill. According to one set of auxiliary assumptions, the received view predicts that desire attributions for failure should be more negative in the high skill than low skill conditions but desire attributions for success should be greater in the low skill than high skill conditions. According to a different set of auxiliary assumptions, the received view predicts that the attenuation effect obtained with intentions also should occur with desires but possibly in an even more exaggerated way. In contrast, according to the confluence theory, there should be little attenuation in the low skill condition, and so the interaction should be weaker. In addition, to the small extent that there is an effect, it should be one where high skill implies more positive desire in the event of success and more negative desire in the event of failure, compared to low skill.

\section{Experiment 1}

\subsection{Methods}

\subsubsection{Participants}

Thirty-seven (21 females) undergraduate students from the New Mexico State University community participated in the experiment for partial course credit. The mean age was 22.13 $(\mathrm{SD}=7.14)$.

\subsubsection{Materials and Stimuli}

The experiment was presented via SurveyMonkey on a $2.0 \mathrm{GHz}$ Dell personal computer with a 21 " monitor that had $1024 \times 768$ resolution.

\subsubsection{Procedure}

Participants first filled out a consent form and were seated comfortably in front of the experimental display. Because the competing predictions of the different viewpoints involved 


\section{Macrothink Institute ${ }^{\mathrm{TM}}$}

the interaction of skill and outcome, it was important to devise scenarios where both could be manipulated in a way that would be plausible to the participants. Consequently, we presented participants with a short scenario about someone who was involved in an activity requiring skill (basketball, stock picking, taking a driver's exam, or performing surgery). The following is an example scenario. Jackie is a basketball player. The game is on the line, and she needs to make a free throw to win the game. Participants were then told that Jackie was an excellent or poor free-throw shooter, and that she either made or missed the shot $(2 \times 2$ within-participants design). Finally, participants were asked to determine "To what extent did [name] intend the actual outcome (making or missing the free-throws)?" Participants provided ratings on a 7-point scale from "extremely unintended" to "extremely intended."

\subsection{Results}

The results are divided into four sections, depending on the type of activity in which the person was involved. Data are presented in Figure 1.

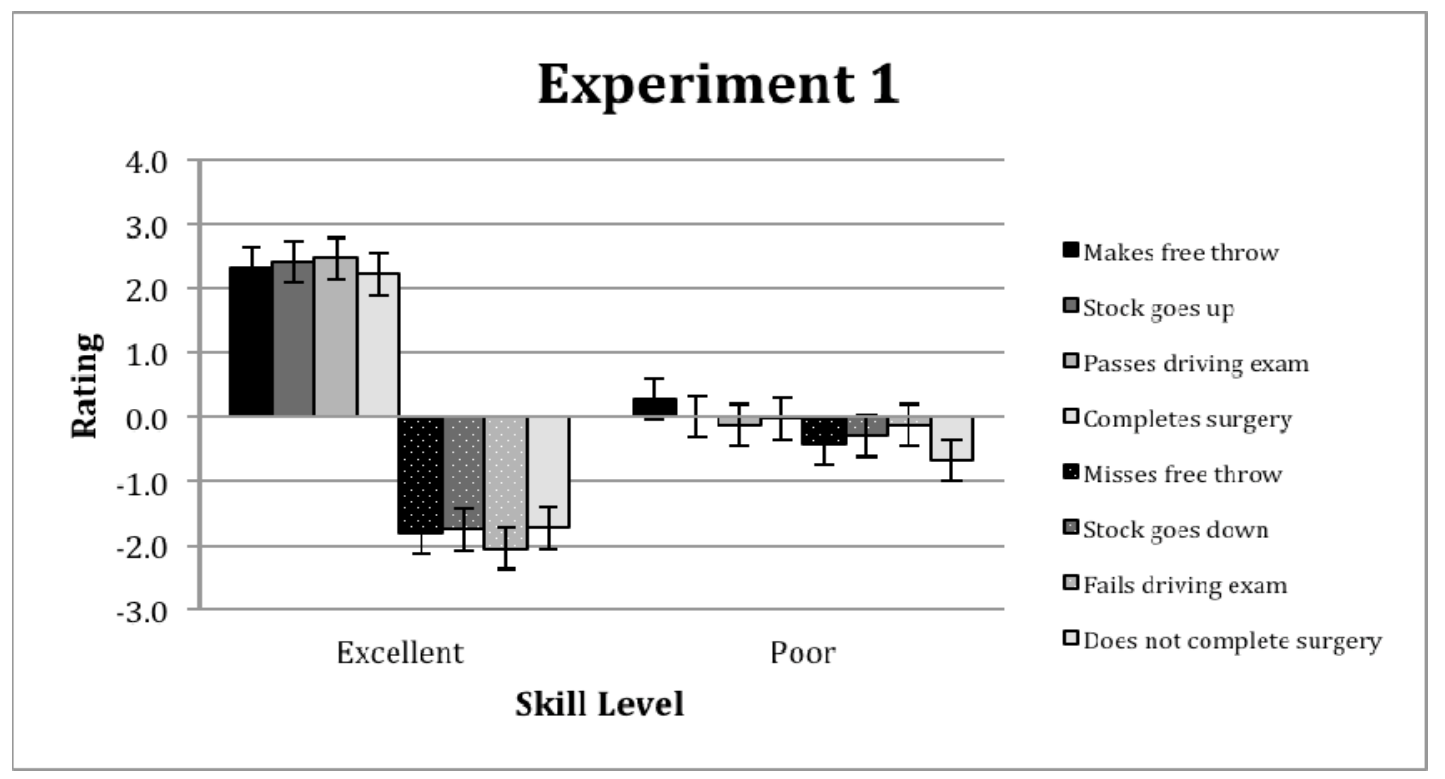

Figure 1. Intention attributions from Experiment 1 as a function of Skill and Outcome. SE bars are included

\subsubsection{Basketball}

A two-way ANOVA using Skill and Outcome as the two factors revealed a main effect of Skill, $F(1,36)=4.47, p<.05, \quad p^{2}=.11$, a main effect of Outcome, $F(1,36)=39.21, p$ $<.001, p^{2}=.52$, and a significant interaction between Skill and Outcome, $F(1,36)=24.33$, $p<.001, \quad p^{2}=.40$.

\subsubsection{Stock Picking}

A two-way ANOVA using Skill and Outcome as the two factors revealed a main effect of Skill, $F(1,36)=9.18, p<.01,{ }_{p}^{2}=.20$, a main effect of Outcome, $F(1,36)=33.99, p<.001$, 


\section{Macrothink}

$p^{2}=.49$, and a significant interaction between Skill and Outcome, $F(1,36)=26.10, p<.001$, $p^{2}=.42$.

\subsubsection{Driver's Exam}

A two-way ANOVA using Skill and Outcome as the two factors revealed no main effect of Skill, $F(1,36)=2.49, p>.10, p^{2}=.07$. There was a main effect of Outcome, $F(1,36)=30.45$, $p<.001,{ }_{p}^{2}=.46$, and a significant interaction between Skill and Outcome, $F(1,36)=34.47$, $p<.001, p^{2}=.49$.

\subsubsection{Surgery}

A two-way ANOVA using Skill and Outcome as the two factors revealed a main effect of Skill, $F(1,36)=5.89, p<.05,{ }_{p}{ }^{2}=.14$, a main effect of Outcome, $F(1,36)=25.77, p<.001$, $p^{2}=.42$, and a significant interaction between Skill and Outcome, $F(1,36)=18.15, p<.001$, $p^{2}=.34$.

\section{Experiment 2}

\subsection{Methods}

It is possible that idiosyncratic aspects of the scenarios we used in Experiment 1 were responsible for the findings. It also is possible that the way the intention attribution measure was worded had an effect. We changed the scenarios and the wording of the dependent variable to address these issues.

\subsubsection{Participants}

Forty-three (28 females) undergraduate students from the New Mexico State University community participated in the experiment for partial course credit. The mean age was 20.46 $(S D=7.98)$.

\subsubsection{Procedure}

Experiment 2 was identical to Experiment 1 with two exceptions. Participants were presented with three different short scenarios about someone completing a task (solving a Rubik's Cube puzzle, finishing a $10-\mathrm{K}$ race, and reading a long book), and this time, they were asked to determine "To what extent did [name] intend to solve the Rubik's Cube?" Participants provided ratings on a 7-point scale from "extremely unintended" to "extremely intended."

\subsection{Results}

Data are presented in Figure 2. 


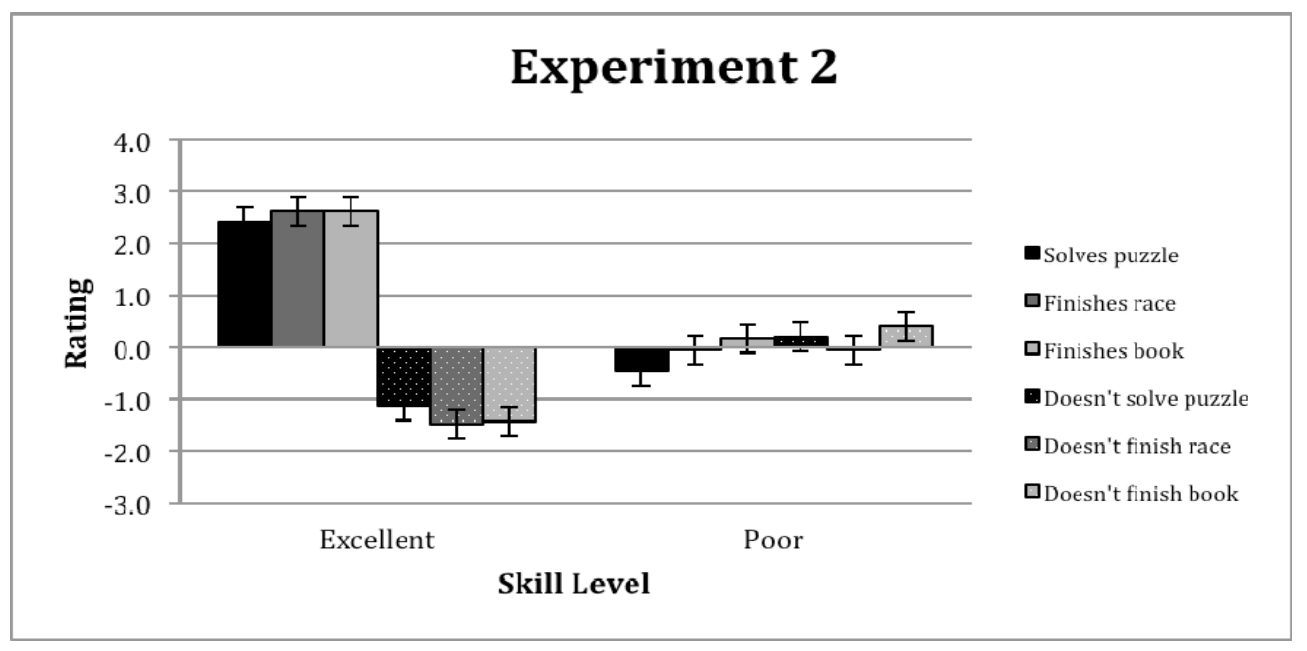

Figure 2. Intention attributions from Experiment 2 as a function of Skill and Outcome. SE bars are included

\subsubsection{Rubik's Cube}

A two-way ANOVA using Skill and Outcome as the two factors revealed a main effect of Skill, $F(1,42)=10.04, p<.01,{ }_{p}{ }^{2}=.19$, a main effect of Outcome, $F(1,42)=20.96, p<.001$, $p^{2}=.33$, and a significant interaction between Skill and Outcome, $F(1,42)=32.82, p<.001$, $p^{2}=.44$.

\subsubsection{0-K Race}

A two-way ANOVA using Skill and Outcome as the two factors revealed a main effect of Skill, $F(1,42)=5.58, p<.05,{ }_{p}{ }^{2}=.12$, a main effect of Outcome, $F(1,42)=39.01, p<.001$, $p^{2}=.48$, and a significant interaction between Skill and Outcome, $F(1,42)=31.34, p<.001$, $p^{2}=.43$.

\subsubsection{Read Long Book}

A two-way ANOVA using Skill and Outcome as the two factors revealed no main effect of Skill, $F(1,42)=1.89, p>.10,{ }_{p}{ }^{2}=.04$. There was a main effect of Outcome, $F(1,42)=37.78$, $p<.001,{ }_{p}^{2}=.47$, and a significant interaction between Skill and Outcome, $F(1,42)=35.08$, $p<.001,{ }_{p}^{2}=.46$.

\section{Discussion-Experiments 1 and 2}

Consistent with predictions from all perspectives, the effect of success versus failure on intentions in the high skill condition attenuates significantly in the low skill condition. However, different predictions arise with respect to desire attributions. These predictions are tested in Experiments 3 and 4. 


\section{Macrothink}

\section{Experiment 3}

\subsection{Method}

\subsubsection{Participants}

Twenty undergraduate students (9 females) from the New Mexico State University community participated in the experiment for partial course credit. The mean age was 21.75 $(S D=8.03)$.

\subsubsection{Procedure}

Experiment 3 was identical to Experiment 1 with one exception. Participants were asked to determine "To what extent did [name] desire the actual outcome (making or missing the free-throws)?" Participants provided ratings on a 7-point scale from "extremely undesired" to "extremely desired."

\subsection{Results}

Data are presented in Figure 3.

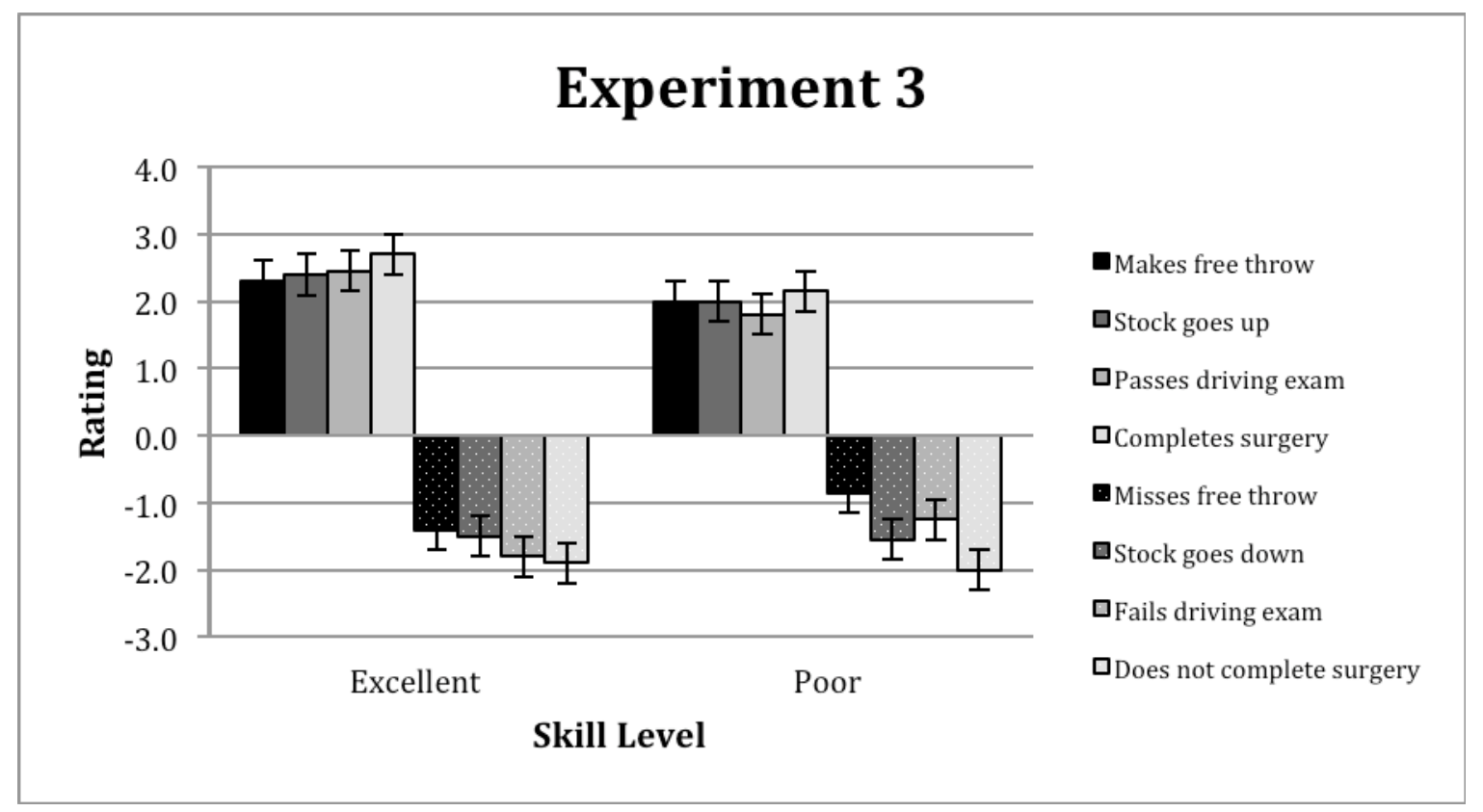

Figure 3. Desire attributions from Experiment 3 as a function of Skill and Outcome. SE bars are included

\subsubsection{Basketball}

Two-way ANOVA using Skill and Outcome as the two factors revealed no main effect of Skill, $F(1,19)=0.09, p>.10,{ }_{p}{ }^{2}=.01$. There was a main effect of Outcome, $F(1,19)=70.59$, $p<.001, p^{2}=.79$. There was no significant interaction between Skill and Outcome, $F(1,19)=$ $2.90, p>.10, p^{2}=.13$. 


\subsubsection{Stock Picking}

A two-way ANOVA using Skill and Outcome as the two factors revealed no main effect of Skill, $F(1,19)=1.19, p>.10,{ }_{p}{ }^{2}=.06$. There was a main effect of Outcome, $F(1,19)=80.21$, $p<.001,{ }_{p}{ }^{2}=.81$. There was no significant interaction between Skill and Outcome, $F(1,19)=$ $0.54, p>.10,{ }_{p}^{2}=.03$.

\subsubsection{Driver's Exam}

A two-way ANOVA using Skill and Outcome as the two factors revealed no main effect of Skill, $F(1,19)=0.04, p>.10,{ }^{2}=.00$. There was a main effect of Outcome, $F(1,19)=94.54$, $p<.001,{ }^{2}=.83$. There was no significant interaction between Skill and Outcome, $F(1,19)=$ $3.62, p>.05,{ }_{p}^{2}=.16$.

\subsubsection{Surgery}

A two-way ANOVA using Skill and Outcome as the two factors revealed no main effect of Skill, $F(1,19)=1.99, p>.10,{ }_{p}^{2}=.09$. There was a main effect of Outcome, $F(1,19)=$ 112.88, $p<.001,{ }_{p}^{2}=.86$. There was no significant interaction between Skill and Outcome, $F(1,19)=1.26, p>.10,{ }_{p}^{2}=.06$.

\section{Experiment 4}

\subsection{Method}

Experiment 2 was performed to address issues pertaining to possible idiosyncrasies of the scenarios or wording of the dependent variable in Experiment 1. In a parallel vein, Experiment 4 was performed to address similar issues stemming from Experiment 3.

\subsubsection{Participants}

Nineteen undergraduate students (10 females) from the New Mexico State University community participated in the experiment for partial course credit. The mean age was 21.47 $(S D=8.24)$.

\subsubsection{Procedure}

Experiment 4 was identical to Experiment 2 with one exception. Participants were asked to determine "To what extent did [name] desire to solve the Rubik's Cube?" Participants provided ratings on a 7-point scale from "extremely undesired" to "extremely desired."

\subsection{Results}

Data are presented in Figure 4. 


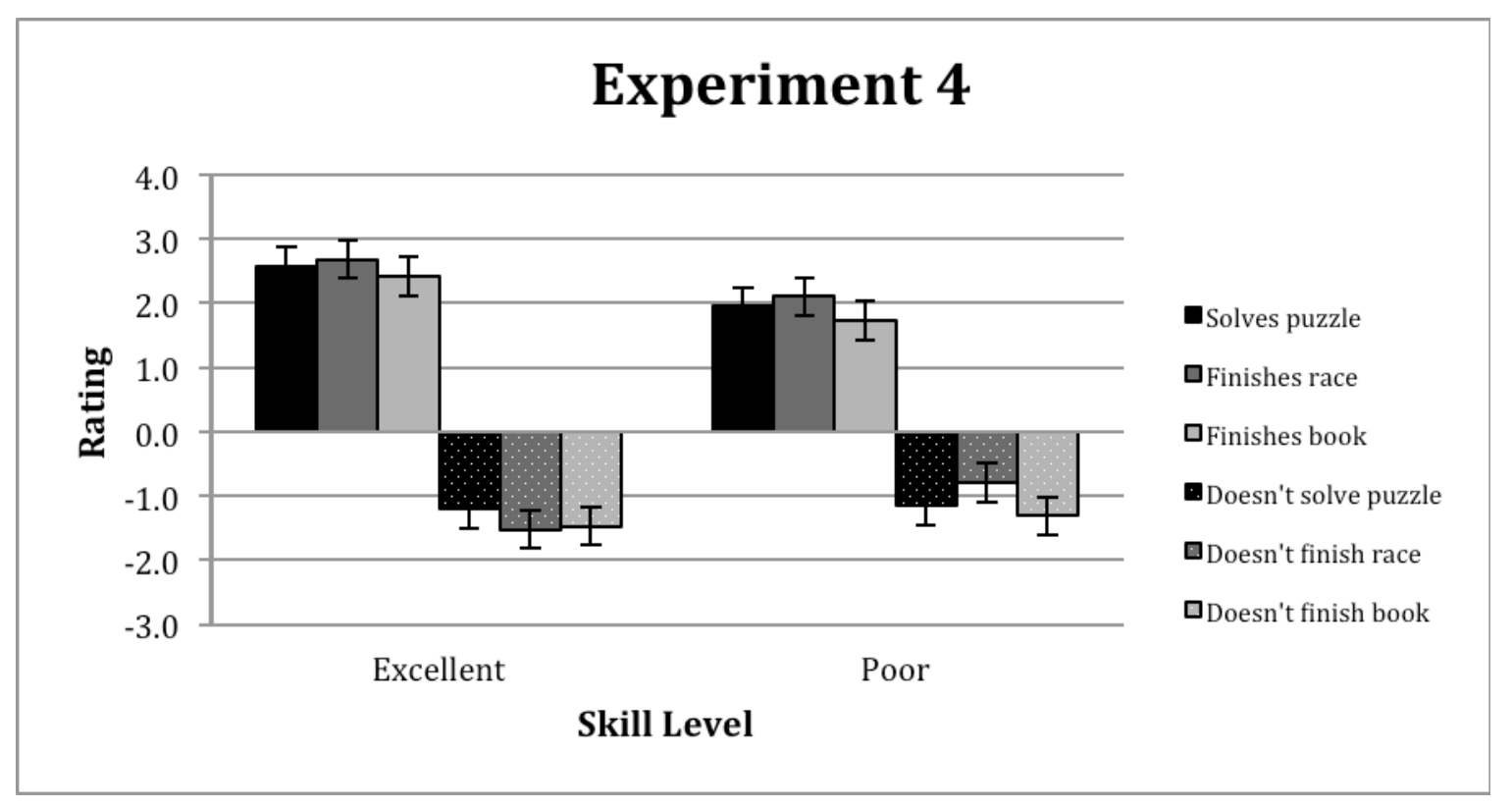

Figure 4. Desire attributions from Experiment 4 as a function of Skill and Outcome. SE bars are included

\subsubsection{Rubik's Cube}

A two-way ANOVA using Skill and Outcome as the two factors revealed no main effect of Skill, $F(1,18)=1.24, p>.10,{ }^{2}=.06$. There was a main effect of Outcome, $F(1,18)=99.26$, $p<.001, p^{2}=.85$. There was no significant interaction between Skill and Outcome, $F(1,18)=$ $1.70, p>.10, p^{2}=.09$.

\subsubsection{0-K Race}

A two-way ANOVA using Skill and Outcome as the two factors revealed no main effect of Skill, $F(1,18)=0.08, p>.10,{ }_{p}{ }^{2}=.00$. There was a main effect of Outcome, $F(1,18)=89.10$, $p<.001,{ }_{p}^{2}=.83$, and a significant interaction between Skill and Outcome, $F(1,18)=12.31$, $p<.01,{ }_{p}^{2}=.41$.

\subsubsection{Read Long Book}

A two-way ANOVA using Skill and Outcome as the two factors revealed no main effect of Skill, $F(1,18)=0.14, p>.10,{ }_{p}{ }^{2}=.01$. There was a main effect of Outcome, $F(1,18)=85.70$, $p<.001,{ }^{2}=.83$. There was no significant interaction between Skill and Outcome, $F(1,18)=$ $0.14, p>.10, p^{2}=.01$.

\section{Discussion-Experiments 3 and 4}

The attenuation effect for intentions in the poor skill condition of Experiments 1 and 2 was reduced to the point of non-significance for desires in Experiments 3 and 4. The one exception was the $10-\mathrm{K}$ race scenario, where significant attenuation occurred even for desire attributions. In addition, across the seven scenarios in Experiments 3 and 4, the slight 
difference between the high skill and low skill conditions was due to more extreme positive desire ratings in high skill than low skill conditions in the event of success (7 out of 7) and more extreme negative desire ratings in high skill than low skill conditions in the event of failure (5 out of 7). Although the finding pertaining to failure is consistent with both one of the received view predictions and with the confluence theory prediction, the finding pertaining to successes only is consistent with the prediction from the confluence theory and contradicts all conceivable predictions from the received view. To quantify this in terms of probability, the binomial theorem gives a probability of 7 successes out of 7 trials by chance, assuming a $50 \%$ chance of success on each trial, at .0078 .

Although our main goal of testing different perspectives via their predictions regarding desires already has been achieved, we also wished to test expectations. The typically assumed path to intention is that there is a desire to be fulfilled, there is an expectation that performing a particular behavior will fulfill it, and so the person may form an intention to perform that behavior provided that he or she has the requisite skill. Finally, of course, the behavior may or may not be performed depending partly on other factors. Thus, expectations are someplace between desires and intentions on the typically assumed pathway to behavior. Because expectations are usually considered to be between desires and intentions in the intentionality progression, we expected the findings to be somewhere in between those obtained in the intention attribution experiments and the desire attribution experiments. That is, we expected the attenuation effect for expectation attributions to be larger than for desire attributions but smaller than for intention attributions.

\section{Experiment 5}

\subsection{Method}

\subsubsection{Participants}

Fifty-seven undergraduate students (29 females) from the New Mexico State University community participated in the experiment for partial course credit. The mean age was 20.19 $(S D=3.96)$.

\subsubsection{Procedure}

Experiment 5 was identical to Experiment 1 with one exception. Participants were asked to determine "To what extent did [name] expect the actual outcome (making or missing the free-throws)?" Participants provided ratings on a 7-point scale from "extremely unexpected" to "extremely expected".

\subsection{Results}

Data are presented in Figure 5. 


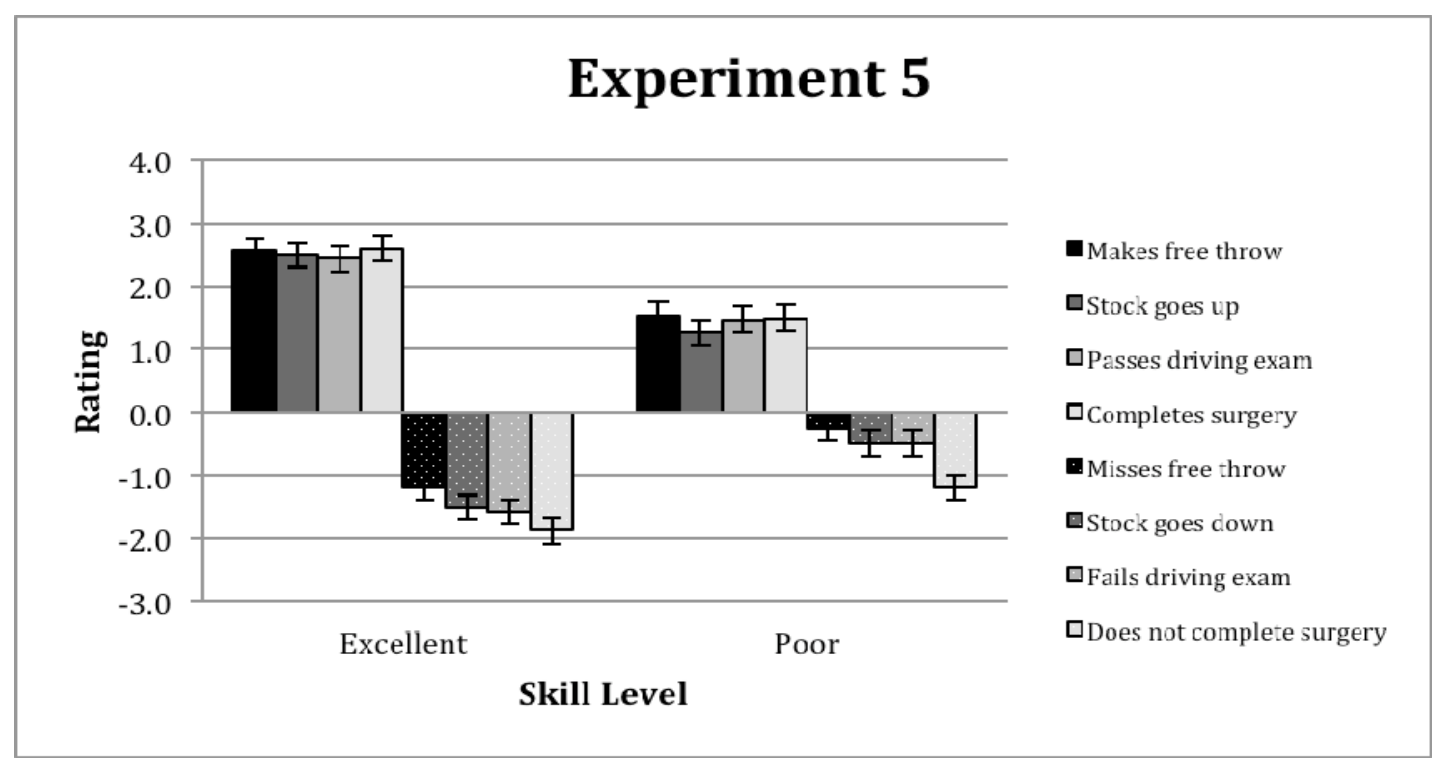

Figure 5. Expectation attributions from Experiment 5 as a function of Skill and Outcome. SE bars are included

\subsubsection{Basketball}

A two-way ANOVA using Skill and Outcome as the two factors revealed no main effect of Skill, $F(1,56)=0.04, p>.10,{ }^{2}=.00$. There was a main effect of Outcome, $F(1,56)=81.86$, $p<.001,{ }_{p}^{2}=.59$, and a significant interaction between Skill and Outcome, $F(1,56)=16.43$, $p<.001, p^{2}=.23$.

\subsubsection{Stock Picking}

A two-way ANOVA using Skill and Outcome as the two factors revealed no main effect of Skill, $F(1,56)=0.35, p>.10,{ }_{p}{ }^{2}=.01$. There was a main effect of Outcome, $F(1,56)=88.48$, $p<.001,{ }_{p}^{2}=.61$, and a significant interaction between Skill and Outcome, $F(1,56)=29.03$, $p<.001,{ }_{p}^{2}=.24$.

\subsubsection{Driver's Exam}

A two-way ANOVA using Skill and Outcome as the two factors revealed no main effect of Skill, $F(1,56)=0.09, p>.10,{ }_{p}^{2}=.00$. There was a main effect of Outcome, $F(1,56)=$ $121.67, p<.001, p^{2}=.69$, and a significant interaction between Skill and Outcome, $F(1,56)=$ $16.51, p<.001,{ }_{p}^{2}=.23$.

\subsubsection{Surgery}

A two-way ANOVA using Skill and Outcome as the two factors revealed no main effect of Skill, $F(1,56)=1.45, p>.10,{ }_{p}^{2}=.03$. There was a main effect of Outcome, $F(1,56)=$ $162.96, p<.001,{ }_{p}^{2}=.74$, and a significant interaction between Skill and Outcome, $F(1,56)=$ $17.58, p<.001,{ }^{2}=.24$. 


\section{Ml Macrothink}

\section{Experiment 6}

\subsection{Method}

Experiment 6 parallels Experiments 2 and 4, but using an expectation measure.

\subsubsection{Participants}

Sixty-three undergraduate students (31 females) from the New Mexico State University community participated in the experiment for partial course credit. The mean age was 20.02 $(S D=3.80)$.

\subsubsection{Procedure}

Experiment 6 was identical to Experiment 2 with one exception. Participants were asked to determine "To what extent did [name] expect to solve the Rubik's Cube?" Participants provided ratings on a 7-point scale from "extremely unexpected" to "extremely expected."

\subsection{Results}

Data are presented in Figure 6.

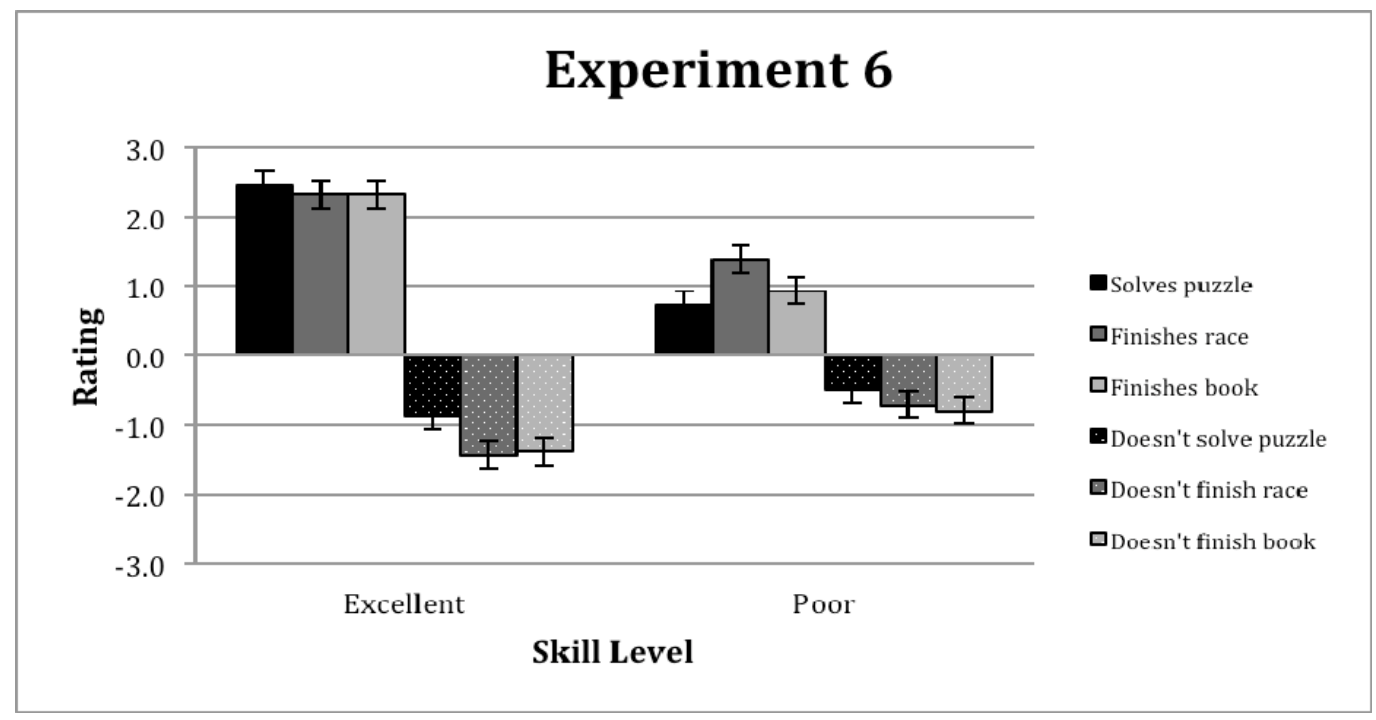

Figure 6. Expectation attributions from Experiment 6 as a function of Skill and Outcome. SE bars are included

\subsubsection{Rubik's Cube}

A two-way ANOVA using Skill and Outcome as the two factors revealed a main effect of Skill, $F(1,62)=6.02, p<.05,{ }_{p}{ }^{2}=.09$, a main effect of Outcome, $F(1,62)=78.95, p<.001$, $p^{2}=.56$, and a significant interaction between Skill and Outcome, $F(1,62)=18.14, p<.001$, $p^{2}=.23$. 


\subsubsection{0-K Race}

A two-way ANOVA using Skill and Outcome as the two factors revealed no main effect of Skill, $F(1,62)=0.16, p>.10,{ }_{p}{ }^{2}=.00$. There was a main effect of Outcome, $F(1,62)=$ 144.17, $p<.001,{ }^{2}=.70$, and a significant interaction between Skill and Outcome, $F(1,62)=$ $12.52, p<.01,{ }_{p}^{2}=.17$.

\subsubsection{Read Long Book}

A two-way ANOVA using Skill and Outcome as the two factors revealed no main effect of Skill, $F(1,62)=1.93, p>.10,{ }_{p}{ }^{2}=.03$. There was a main effect of Outcome, $F(1,62)=$ $111.17, p<.001, p^{2}=.64$, and a significant interaction between Skill and Outcome, $F(1,62)=$ $16.97, p<.001,{ }_{p}^{2}=.22$.

\section{Discussion-Experiments 5 and 6}

According to the confluence theory, the attenuation effect was supposed to be largest for intentions, to mostly disappear for desires, and to be somewhere in between for expectations. In fact, this is what happened. Consider the central tendencies for interaction partial eta squares across the scenarios for intentions $(M=.43$ and $M d=.43)$, desires $(M=.13$ and $M d$ $=.09)$, and expectations $(M=.22$ and $M d=.23)$. For a statistical test across experiments, consider that there were 7 scenarios (four in Experiments 1, 3, and 5 and three in Experiments 2, 4, and 6), and so there were three comparisons of effect sizes to be made within each scenario across the three dependent measures (intentions, desires, and expectations), for a total of $7 \times 3=21$ effect size comparisons. Out of these 21 comparisons, 20 were in the direction predicted by our confluence perspective (Binomial Test $p<.001$ ).

Alternatively, consider the difference between success and failure in the attenuation (poor skill) condition, where the confluence theory predicts the smallest differences for intentions (greatest attenuation) and the largest ones for desires (least attenuation). The central tendencies of these differences across the scenarios are consistent with predictions for intentions $(M=.40$ and $M d=.30)$, desires $(M=3.24$ and $M d=3.06)$, and expectations $(M=$ 1.89 and $M d=1.80)$. Examining across scenarios and across dependent measures, as in the foregoing paragraph, results in similar support for our confluence perspective (Binomial Test $p<.001)$.

\section{General Discussion}

We have seen that competing predictions pertaining to desires can be made from several different points of view. According to one way of thinking, unskilled people have to try harder to succeed than do skilled people. Consequently, success implies more desire in unskilled people than in skilled people.

According to another way of thinking, desire is relevant to outcomes only for skilled people but not for unskilled people. Therefore, although success or failure should have a large effect on desire attributions pertaining to skilled persons, there should be practically no effect on desire attributions pertaining to unskilled persons. The data strongly contradicted both of these points of view. 
The notion of a tug-of-war between confluence and counterforces suggests a third way of thinking. The idea here, put simply, is that success engulfs the field unless there is a counterforce. In the case of intentions, there is a counterforce and so people who are not skilled but succeed anyway are thought to have lower intentions than skilled people who are successful. But unlike intention attributions, low skill does not provide a counteracting force for desire attributions. Hence, success or failure has a large effect regardless of skill. But the confluence theory makes yet a further prediction. Because the three-way combination of success, high skill, and high desire provides for more overall compatibility than the three-way combination of success, low skill, and high desire, desire attributions for success should be greater, rather than less, in the high skill condition relative to the low skill condition. The findings were consistent with all of these predictions. Finally, because expectations are usually considered to be between desires and intentions in the intentionality progression, we expected, and obtained, an intermediate effect on expectation attributions, between that obtained with intention and desire attributions.

\subsection{Potential Criticisms}

As is generally so, the present research has limitations. One limitation is that the research depended on scenarios and perhaps the results were due to idiosyncratic aspects of our scenarios. There can be little doubt that this danger always exists in research involving scenarios. However, we believe that there are factors that mitigate the danger. First, we used seven different scenarios. It is not plausible that we just happened to pick the right seven scenarios to make confluence theory predictions work out. In addition, practically all of the research in this area is performed with scenarios. Had we not used them, the criticism would be that our findings would have been unable to be compared to those obtained by previous researchers. Nevertheless, we recognize that the extent to which our findings would generalize to the scenarios people experience by actually living in them is currently unclear. Therefore, our research is better characterized as theory testing than as concerning generalization of findings.

A second limitation is that we used fewer participants in the experiments involving desires than intentions or expectations. Arguably, the lack of significant interactions in those experiments could have been due to an insufficient number of participants. The problem with this potential criticism is that it does not take effect sizes into account. The number of participants does not influence the expected values of sample effect sizes. And yet, as we showed, comparing the interaction effect sizes within each scenario, across dependent variables (intentions, desires, and expectations), resulted in 20 out of 21 comparisons being in the direction predicted by our confluence perspective. We obtained similar support for our confluence perspective even when we confined the analyses to the attenuation condition (poor skill). Therefore, although the differences in sample sizes across experiments can be considered to be a limitation, it in no way compromises our conclusions.

A third limitation is that we used different dependent variables in different experiments that involved different participants, rather than including them in the same experiment. However, because the participants in all experiments were sampled from the same population of 
introductory psychology students, we believe it is difficult to attribute our effects to using different dependent variables in different experiments. We recognize, of course, that this might limit the generalizability of our findings to other populations, but we feel it is not an important problem for our goal of testing the confluence theory. In fact, consider the counterfactual where the same participants would have received all of the dependent variables in the same experiment. In that case, the criticism would be that completing one of the dependent variables might have influenced the completion of the other ones. This would have been an important criticism that would have compromised our conclusions. Fortunately, it was easy to avoid, as we did, by simply using different dependent variables in different experiments.

\subsection{General Implications}

The present research differed from other research in the attribution area in at least two respects. One difference is that, in addition to testing for intention attributions, we also tested desire and expectation attributions. Given that desires and expectations are considered to be necessary conditions for intentions, it makes sense for attribution articles to consider them in conjunction with each other rather than separately. We have seen that there seems to be a rather orderly empirical progression among attributions pertaining to these three variables. That is, the attenuation effect, whereby success or failure strongly influences attributions in high skill conditions but does so to a lesser degree in low skill conditions, varies in strength depending on the type of attribution of concern. The attenuation effect is weakest for desire attributions, stronger for expectation attributions, and stronger yet for intention attributions.

This empirical continuation dovetails nicely with the process that often is thought to lead to behavior. As we suggested earlier, people believe that others perform behaviors because of some desire to be fulfilled, which leads to an expectation that the performance of a behavior will lead to fulfillment of the desire, which leads to an intention to perform the behavior. Finally, of course, there is the actual behavior. Thus, the attenuation effect is weakest for attributions pertaining to the variable most distal from the behavior (desire), stronger for attributions pertaining to a more proximal variable (expectations), and even stronger for attributions pertaining to a yet more proximal variable (intentions). The progression of increasing attenuation effects as attributions progress from the variable most distal to behavior to the variable most proximal to behavior is unlikely to be a coincidence. Even if it eventually turns out that the confluence theory is wrong - a risk that one always must consider when deriving predictions from theories - the empirical discovery of a progression of attenuation effects is likely to inform any theory that replaces it.

\subsection{Conclusion}

Most theories in psychology are mechanistic. That is, they specify a causal chain that can be tested via experiment. Confluence theory is an exception; it does not specify a specific causal mechanism. Rather, it is more of a unifying notion than a causal one. Although unification may be out of fashion in social psychology, we believe that there is a place for both kinds of theories in a science of social psychology. The philosopher Salmon (1998) provides a nice illustration of the value of unifying theories in physics that we paraphrase below. 
Imagine that one has a helium filled balloon on a string while sitting in a seat on a jet that is taking off. While the jet is accelerating forward, what will happen to the helium filled balloon - will it move forward (towards the front of the jet) or backward (towards the rear of the jet)? The answer is that it will move forward, but there are two classes of explanations for the balloon's behavior. A mechanism explanation is that, upon accelerating, the rear wall of the jet exerts forward force on the adjacent air molecules which, in turn, move more air molecules in that direction, and so on. Because the helium filled balloon is less dense than the air, it is forced forward. Alternatively, a more unification type of explanation invokes Einstein's theory of relativity. To see this, consider that according to Einstein's theory, acceleration and gravity are indistinguishable. As the plane accelerates forward, that is equivalent to generating a "gravitational" force towards the rear of the jet, and because the helium filled balloon is less dense than air, it "floats" relative to the "heavier" air molecules, which is equivalent to its moving towards the front of the jet in the direction opposite to the gravitational force generated by the jet's acceleration.

Salmon's point with this illustration is not that one of the theories is a bad theory but rather that the theories are at different levels, with the former being more mechanistic and the latter being more unifying. With Salmon, we believe that both types of theories are desirable to have in a scientific discipline. But there are relatively few unifying theories in social psychology, so it is, perhaps, time to redress the balance. We hope and expect that the confluence theory will provide an impetus in this direction.

\section{References}

Ames, D. R. (2009). Reframing the rocky road: From causal analysis to mindreading as the drama of disposition inference. Psychological Inquiry, 20, 19-23. http://dx.doi.org/10.1080/10478400902744253

Edwards, W. (1954). The theory of decision making. Psychological Bulletin, 51, 380-417. http://dx.doi.org/10.1037/h0053870

Fishbein, M. (1963). An investigation of the relationships between beliefs about an object and the attitude toward that object. Human Relations, 16, 233-239. http://dx.doi.org/10.1177/001872676301600302

Fishbein, M., \& Ajzen, I. (1975). Belief, attitude, intention and behavior: An introduction to theory and research. Reading, MA: Addison-Wesley.

Malle, B. F. (1999). How people explain behavior: A new theoretical framework. Personality and Social Psychology Review, 3, 23-48. http://dx.doi.org/10.1207/s15327957pspr0301_2

Malle, B. F. (2004). How the mind explains behavior: Folk explanations, meaning, and social interaction. Cambridge, MA: MIT Press.

Molden, D. C. (2009). Finding meaning in others' intentions: The process of judging intentional behaviors and intentionality itself. Psychological Inquiry, 20, 37-43. http://dx.doi.org/10.1080/10478400902744295

Morris, M. W. (2009). Intentionality in intuitive versus analytic processing: Insights from 
social cognitive neuroscience. Psychological Inquiry, 20, 58-65. http://dx.doi.org/10.1080/10478400902794548

Newman, L. S. (2009). Have dispositional inferences been oversold? The role of trait judgments in the Multiple Inference Model. Psychological Inquiry, 20, 44-47. http://dx.doi.org/10.1080/10478400902744303

Peak, H. (1958). Psychological structure and psychological activity. Psychological Review, 6, 325-347. http://dx.doi.org/10.1037/h0048404

Reeder, G. D. (2009a). Mindreading: Judgments about intentionality and motives in $\begin{array}{lllll}\text { dispositional inference. Psychological } & \text { Inquiry, } & \text { 20, } & \text { 1-18. }\end{array}$ http://dx.doi.org/10.1080/10478400802615744

Reeder, G. D. (2009b). Mindreading and dispositional inference: MIM revised and extended. Psychological Inquiry, 20, 73-83. http://dx.doi.org/10.1080/10478400902868565

Reeder, G. D., Hesson-McInnis, M., Krohse, J. O., \& Scialabba, E. A. (2001). Inferences about effort and ability. Personality and Social Psychology Bulletin, 27, 1225-1235. http://dx.doi.org/10.1177/0146167201279014

Rice, S. \& Trafimow, D. (2011). It's a just world no matter which way you look at it. Journal of General Psychology, 138(3), 229-242. http://dx.doi.org/10.1080/00221309.2011.582524

Rice, S., Trafimow, D., Keller, D. \& Bean, N. (in press). Confluence Theory: Uniting two houses divided. Theoretical Issues in Ergonomics Science. http://dx.doi.org/10.1080/1463922x.2010.525760

Rosenberg, M. J. (1956). Cognitive structure and attitudinal affect. Journal of Abnormal and Social Psychology, 53, 367-372. http://dx.doi.org/10.1037/h0044579

Rotter, J. (1954). Social learning and clinical psychology. New York: Prentice-Hall, Inc. http://dx.doi.org/10.1037/10788-000

Salmon, W. C. (1998). Causality and Explanation. Oxford: Oxford University Press. http://dx.doi.org/10.1093/0195108647.001.0001

Trafimow, D. (2009). Reeder's MIM as a special case of confluence theory. Psychological Inquiry, 20, 48-52. http://dx.doi.org/10.1080/10478400902744311

Trafimow, D., Rice, S., Hunt, G., List, B., Nanez, B., Rector, N., ... Brown, J. (in press). It's irrelevant, but it matters: Using Confluence Theory to predict the influence of beliefs on evaluations, attitudes and intentions. European Journal of Social Psychology.

\section{Copyright Disclaimer}

Copyright reserved by the author(s).

This article is an open-access article distributed under the terms and conditions of the Creative Commons Attribution license (http://creativecommons.org/licenses/by/3.0/). 